\title{
A Giant Sumucosal Lipoma of Large Intestine Presenting With Intussusception in An Adult: A Case Report.
}

\author{
Dr. Ridhima ${ }^{1}$,Dr. (Mrs.) Sharda Dawan ${ }^{2}$, Dr. (Mrs.) Vanita Kumar ${ }^{3}$, \\ Dr. (Mrs.) Neelu Gupta ${ }^{4}$ \\ 1,23,4 (Department Of Pathology, Sardar Patel Medical College\& A.G. Hospitals, Bikaner/Rajasthan University Of \\ Health Sciences, India)
}

\begin{abstract}
Introduction: Colon lipomas are rare. They are almost always asymptomatic; only when their diameter is more than $3 \mathrm{~cm}$ do they become symptomatic. They tend to occur in an older population and most of the cases are located at the right side of the large bowel. Usually the diagnosis is not made until operation is performed. Intussusception is an uncommon cause of intestinal obstruction in adults.

Case presentation: We describe the case of a 52-year-old female who presented with features of intestinal obstruction secondary to intussusception. A colocolonic invagination was found with giant intraluminal lipoma on CECT abdomen. She was treated operatively. Exploratory Laprotomy with Segmental resection of colon wih colocolonic anastamosis was performed. A histopathological examination revealed a polypoid, submucosal lipoma.

Conclusion: In resource-rich countries, most cases of colonic intraluminal polyps are detected through colonoscopy during routine medical check-ups. With limited resources in our region, many tumors present as intestinal obstructions secondary to intussusception. Even then, most are associated with adenomas and malignancies. Rarely are polypoid, submucosal lipomas found. In our patient's case a polyp of $4.6 \mathrm{~cm}$ at its widest dimension is of particular interest.
\end{abstract}

Keywords: Colocolonic, giant polyp, intestinal obstruction, intraluminal, intussusception, lipoma, submucosal

\section{Introduction}

Lipoma of the gastrointestinal tract is a rare benign condition described for the first time in 1757 by Baurer and reported in only $0.2 \%-4.4 \%$ of large autopsy series since $1955^{[1]}$. The symptomatic patients with lipomas present with abdominal pain, haemorrhage, diarrhoea, or constipation ${ }^{[2]}$. Lipomas that grow more than $4 \mathrm{~cm}$ in size can lead to obstruction and intussusception. More commonly, definitive diagnosis is made by histopathological staining after the removal of lipoma ${ }^{[3]}$. Surgical resection is recommended to alleviate symptoms and to rule out malignancy ${ }^{[3]}$.Intussusception was first described by Barbette of Amsterdam in 1674 ${ }^{[4]}$. It is relatively frequent in children but rare in adults, representing $5 \%$ of all bowel intussusceptions and $1 \%$ of all bowel obstruction. Colonic intussusception is even rarer, above all when caused by lipomas: thirty-seven definite cases have been reported in the English-language literature over the past 45 years ${ }^{[6]}$.In this case report, we describe an intussuscepted, colonic lipoma that caused intermittent bowel obstruction.

\section{Case Report}

A 52-year-old female was admitted to the general surgery department of our hospital for left-sided abdominalpain since 15 days. Her pain was a dull abdominal ache of insidious onset located mainly in the left hypochondrium and lumbar region ; the pain was persistent and was not related to food intake. Associated with this was diffuse abdominal bloating and flatulence with pellet-like stool motions. Her appetite was good and she felt she had neithergained nor lost weight. There was no history of dyspeptic symptoms or vomiting or hematochezia during that time, nor had she felt any swelling in her abdomen. One day prior to admission, she developed mucoid, bloody stool motions, but had no frank diarrhea or distinctively foul-smelling stool. She was also unable to pass flatus. No history of chronic disease was reported; specifically, diabetes mellitus and hypertension The patient was admitted to the hospital and CECT scan of abdomen revealed bowel obstruction with colocolonic intussusception with an intraluminal lipoma. The patient underwent an exploratory laparotomy.

A Segmental resection of colon was performed followed by colocolonic anastomosis and the specimen was sent for histopathological examination.Here in pathology department we received a specimen of intestine measuring $10 \times 7 \times 4 \mathrm{~cm}$.On cutting, a pedunculated polypoidal mass was seen in lumen which measured $4.6 \times 4 \times 2.6 \mathrm{~cm}$ (Fig. 1). The cut surface of mass was glistening yellow and mucosa was lost in the region where it was attached. The microscopic examination confirmed the mass as submucosal lipoma(Fig. 2,3). 

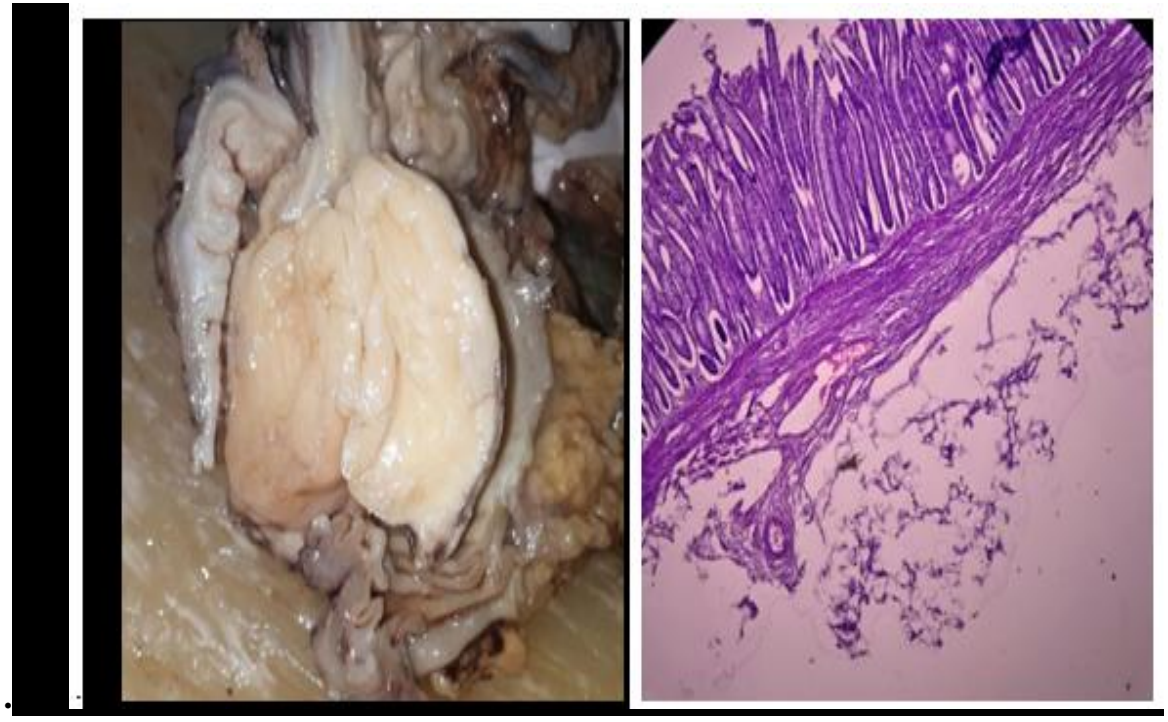

Figure1.Gross examination revealed

Figure 2. Microscopic examination of large glistening yellow polypoidal mass Submucosal lipoma (H\&E, x10)

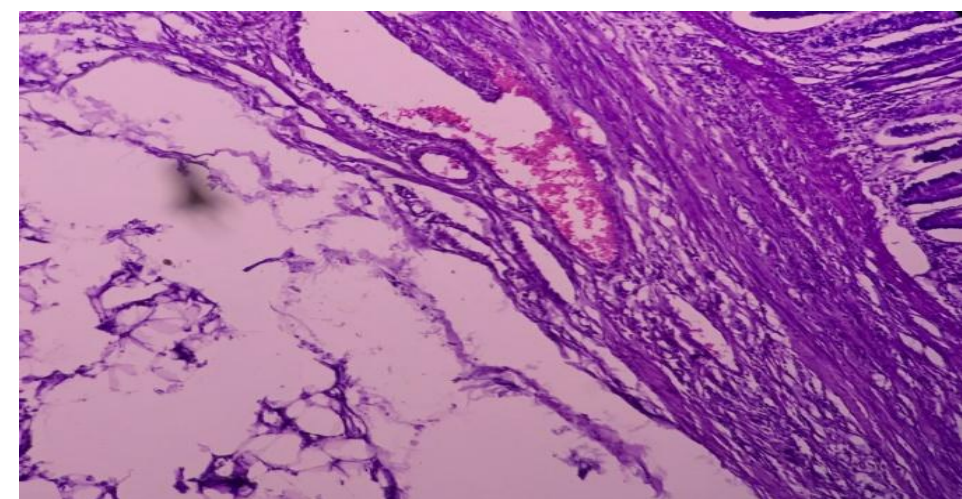

Figure 3. Submucosal lipoma $(H \& E, x 40)$. benign adipocytes in Submucosa with sparing of mucosa

\section{DISCUSSION}

Lipomas occur throughout the intestinal tract with the colon having the highest incidence. They constitute the most frequent benign tumor of the colon and rectum after adenomas. Nevertheless they are uncommon. ${ }^{[7]}$.

Colonic lipomas are more common in women with a peak incidence between 50 and 60 years of age ${ }^{[8,9]}$. They are mostly located in the right colon: $19 \%$ in cecum, $38 \%$ in ascending colon, $22 \%$ in transverse colon, $13 \%$ in the descending colon, and $8 \%$ into the sigmoid colon. The most frequent type is the submucosal lipoma with a sessile or pedunculated appearance, and the remainderare subserosal lipomas. They are almost always asymptomatic; only when their diameter is more than $3 \mathrm{~cm}$ do they become symptomatic. Their clinical symptoms are directly related to their size. The common symptoms of colonic lipomas include changes in intestinal rhythm, rectorrhagia, diarrhea, abdominal pain, occupation of the colonic lumen by the mass, or intestinal intussusception caused by the progression of the pedunculated lipoma ${ }^{[10]}$. After intussusception abdominal pain is associated with vomiting, palpable mass and bloody stool, presenting for

many days or even weeks Lipomas can also present in the form of multiple polyps in the colon. Some may exhipit atypical stromal cells and mimic malignancy. Considering the age and symptoms of these patients, malignant tumors are also a matter of concern ${ }^{[11]}$. For the diagnosis, colonoscopy allows direct visualization of the submucosal lipoma, which appears as a mass covered by normal mucosa, but it can also show ulcerated or necrotic overlying mucosa ${ }^{[6,12,13]}$. Colonoscopic biopsy confirms the nature, but inadequate tissue samples often indicate nonspecific colitis with mucosal inflammation

In case of intussusception, abdominal CT scanning is the radiologic modality of choice, above all when giant lipomas are present, with a $70-80 \%$ sensitivity and near $100 \%$ specificity ${ }^{[5,15]}$. Lipoma appears with fatequivalent density, near ovoidal shape, and smooth margins.However, intussuscepted lipomas may have a heterogeneous appearance reflecting the degree of infarction and fat necrosis ${ }^{[5,16]}$.

There are different options for treatment. Small lipomas, less than $2 \mathrm{~cm}$, can be endoscopically removed. Since lipomas show no malignant degeneration, if the biopsy is unequivocal, they may not need 
treatment and can be observed.Some authors have reported that large pedunculated lesions can be removed without perforation using clipping or endoloop ligation ${ }^{[15]}$, but in most series endoscopic removal of lipomas larger than $2 \mathrm{~cm}$ is associated with a greater risk of perforation ${ }^{[6,15]}$. It is therefore recommended that tumors larger than $2 \mathrm{~cm}$ must be resected surgically Moreover, the size of the lipoma is an essential factor leading to colonic intussusception, particularly when mainaxis of the lesion is over $4 \mathrm{~cm}$. This is the reason why colonic lipomas of $4 \mathrm{~cm}$ or more must be resected before intussusception occurs ${ }^{[6]}$. If a colonic lipoma is diagnosed before surgery, segmental resection is an adequate treatment. Since the majority of colonic intussusceptions are caused by primary adenocarcinoma, in view of the uncertain etiology, nondiagnosed lipoma before operation must be interpreted as for cancer, and a more or less extensive resection of the colon is recommended, depending on the location of the tumor. Patients must undergo more or less extensive resection of the colon also depending on the length of the intussusception segment ${ }^{[6,17]}$

\section{Conclusion}

Lipomas of digestive tract are rare and often asymptomatic and with their unusual properties, present Difficulties in pre-operative differentiation between malignant and benign colonic neoplasms. Furthermore, the investigative procedures are challenging; this is particularly the case in resource-limited areas.. Also, Colonic obstruction due to intussusception caused by lipomas is a very rare condition that needs urgent treatment. CT is the radiologic modality of choice for diagnosis (sensitivity $80 \%$, specificity near $100 \%$ ); since the majority of colonic intussusceptions are caused by primary adenocarcinoma, if the etiology is uncertain, the lesion must be interpreted as malignant and extensive resection is recommended. At present, surgery is the treatment of choice and determines an excellent outcome.

\section{References}

[1]. E. Grasso and T. Guastella, "Giant submucosal lipoma cause colo-colonic intussusception. A case report and review of literature," Annali Italiani di Chirurgia, 83(6), 2012, 559-562.

[2]. Ghidirim G, Mishin I, Gutsu E et al. Giant submucosal lipoma of the cecum: report of a case and review of literature. Romanian Journal of Gastroenterology; 14(4) 2005, 393-6.

[3]. Bardaji M, Roset F, Camps R, Sant F, Fernandez-Layos MJ. Symptomatic colonic lipoma: differential diagnosis of large bowel tumors. International Journal of Colorectal Disease; 13(1), 1998 : 1-2

[4]. A. S. Krasniqi, A. R. Hamza, L. M. Salihu et al., "Compound double ileoileal and ileocecocolic intussusception caused by lipoma of the ileum in an adult patient. A case report," Journal of Medical Case Reports, 5, 2011, 452.

[5]. O. Mouaqit, H. Hasnai, L. Chbani et al., "Pedunculated lipoma causing colo-colonic intussusception: a rare case report," BMC Surgery, 13(1), 2013, 51.

[6]. S. Pa skauskas, T. Latkauskas, G. Valeikaite et al., "Colonic intussusception caused by colonic lipoma: a case report," Medicina, 46( 7), 2010, 477-481.

[7]. Marra B: Intestinal occlusion due to a colonic lipoma. A report of 2 cases. Minerva Chir Sep 30; 48(18): 1993, 1035-9

[8]. R. A. De Beer and H. Shinya, "Colonic lipomas: an endoscopic analysis," Gastrointestinal Endoscopy, 22( 2), 1975, 90-91.

[9]. B. A. Taylor and B. G. Wolff, "Colonic lipomas - Report of two unusual cases and review of the mayo clinic experience, 19761985," Diseases of the Colon \& Rectum, 30(11), 1987, 888-893.

[10]. Cirino E, Cali V, Basile G, Muscari C, Caragliano P, Petino A: Intestinal invagination caused by colonic lipoma. Minerva Chir Sep; 51(9): 1996, 717-23

[11]. Ryan J, Martin JE, Pollock DJ: Fatty tumours of the large intestine: A clinicopathological review of 13 cases. Br J Surg 76: 1989, 793-796.

[12]. M. Michowitz, N. Lazebnik, S. Noy, and R. Lazebnik, "Lipoma of the colon. A report of 22 cases," American Surgeon, 51( 8), 1985, 449-454,

[13]. P. C. Buetow, J. L. Buck, N. J. Carr, L. Pantongrag-Brown, P. R. Ros, and D. F. Cruess, "Intussuscepted colonic lipomas: loss of fat attenuation on CT with pathologic correlation in 10 cases," Abdominal Imaging, 21(2), 1996, 153-156.

[14]. P. Katsinelos, G. Chatzimavroudis, C. Zavos, G. Paroutoglou, B. Papaziogas, and J. Kountouras, "A novel technique for the treatment of a symptomatic giant colonic lipoma," Journal of Laparoendoscopic and Advanced Surgical Techniques, 17(4) , 2007, 467-469.

[15]. G. S. Raju and G. Gomez, "Endoloop ligation of a large colonic lipoma: a novel technique," Gastrointestinal Endoscopy, 62(6), , 2005, 988-990.

[16]. S.Tamura, Y. Yokoyama,T.Morita, T. Tadokoro,Y.Higashidani, and S. Onishi, “'Giant' colon lipoma: what kind of findings are necessary for the indication of endoscopic resection?"American Journal of Gastroenterology, 96(6), 2001, 1944-1946.

[17]. J. Y.-M. Chiang and Y.-S. Lin, “Tumor spectrum of adult intussusception,” Journal of Surgical Oncology, 98(6), $2008,444-447$. 